ARTICLE

\title{
Universal momentum-to-real-space mapping of topological singularities
}

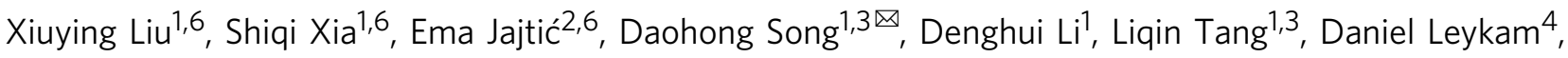 \\ Jingjun Xut, ${ }^{1,3}$, Hrvoje Buljan ${ }^{1,2}$ \& Zhigang Chen (i) ${ }^{1,3,5 凶}$
}

Topological properties of materials are typically presented in momentum space. Here, we demonstrate a universal mapping of topological singularities from momentum to real space. By exciting Dirac-like cones in photonic honeycomb (pseudospin-1/2) and Lieb (pseudospin-1) lattices with vortex beams of topological charge I, optimally aligned with a given pseudospin state $s$, we directly observe topological charge conversion that follows the rule $I \rightarrow I+2 s$. Although the mapping is observed in photonic lattices where pseudospin-orbit interaction takes place, we generalize the theory to show it is the nontrivial Berry phase winding that accounts for the conversion which persists even in systems where angular momentum is not conserved, unveiling its topological origin. Our results have direct impact on other branches of physics and material sciences beyond the 2D photonic platform: equivalent mapping occurs for 3D topological singularities such as Dirac-Weyl synthetic monopoles, achievable in mechanical, acoustic, or ultracold atomic systems, and even with electron beams.

\footnotetext{
${ }^{1}$ The MOE Key Laboratory of Weak-Light Nonlinear Photonics, TEDA Applied Physics Institute and School of Physics, Nankai University, Tianjin 300457 , China. ${ }^{2}$ Department of Physics, Faculty of Science, University of Zagreb, Bijenička c. 32, 10000 Zagreb, Croatia. ${ }^{3}$ Collaborative Innovation Center of Extreme Optics, Shanxi University, Taiyuan, Shanxi 030006, People's Republic of China. ${ }^{4}$ Center for Theoretical Physics of Complex Systems, Institute for Basic Science, Daejeon 34126, Republic of Korea. ${ }^{5}$ Department of Physics and Astronomy, San Francisco State University, San Francisco, CA 94132, USA.

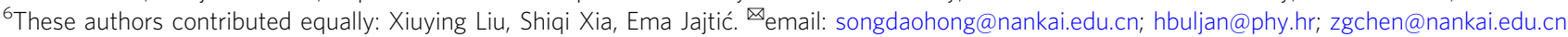


$\mathrm{T}$ opological phases, as manifested in the intriguing phenomena of quantum Hall effect and topological insulators ${ }^{1,2}$, have attracted overwhelming transdisciplinary interest in recent years ${ }^{3-7}$. Topological edge states, for instance, have been realized in a variety of systems including electromagnetic waves ${ }^{8-12}$. Topological properties of Bloch bands are revealed in momentum space, using concepts such as the Chern number and Berry phase. We demonstrate here a universal mapping of the topology of Dirac-like cones from momentum to real space. This is achieved by properly aligned vortex excitation of pseudospin states near the Dirac-like cones in photonic honeycomb (half-integer pseudospin) ${ }^{13,14}$ and Lieb (integer pseudospin $)^{15}$ lattices, leading to direct observation of topological charge conversion (as illustrated in Fig. 1a, b). We develop a unified theory to explain the observed phenomenon and present the mapping in a general topological framework involving nontrivial Berry phase winding. The topological origin of this conversion makes it both robust and universal, persisting in deformed lattices where angular momentum is not conserved, and for 3D Dirac-Weyl synthetic magnetic monopoles ${ }^{16-18}$ (see Fig. 1c), which can be realized in ultracold atomic gases ${ }^{19}$. The underlying mechanism could also be responsible for the vortex creation in electron beams traversing magnetic monopole field ${ }^{20}$.

The coupling of spin and orbital degrees of freedom is in many systems intertwined with the underlying topology of the space and the Berry phase ${ }^{21}$. For instance, in condensed matter electronic systems, studies of spin-orbit interactions led to the discovery of topological insulators, which have now emerged as an important field. The physics of electron beams illustrates many examples where spin-orbit coupling is integrated with topology 22 . There is also a plethora of related examples in optics and photonics $^{23}$ : with real space Berry phase optical elements such as qplates and metasurfaces, circular polarization states of light (intrinsic spin) can be transformed to optical vortices carrying orbital angular momentum (OAM) ${ }^{24-26}$; for light propagating along a coiled ray trajectory, the dynamics is governed by the action of the monopole in Berry curvature, leading to the spinHall effect of light ${ }^{27}$. An analogous topological transport of sound waves has also been observed recently, thanks to the spinredirection geometric phase ${ }^{28}$.

When discussing spin in optical systems, it is the light polarization or photon spin that is usually considered as the spin degree of freedom ${ }^{23,29}$. Similarly, in electronic systems it is the intrinsic electron $\operatorname{spin}^{1,2}$. However, for light (electrons) propagating in structured photonic media (crystalline lattices) with microscopic degrees of freedom, the concept of pseudospin independent of any intrinsic particle property emerges ${ }^{13-15,30-32}$. Such a concept in graphene is introduced through the mathematical analogy between the graphene sublattice degree of freedom and the electron spin in the Dirac equation. Unlike the electron spin, however, the pseudospin angular momentum is not associated with any intrinsic property of particles, but rather arises from the substructure of space (sublattices) that the particles (or wave packets) live in. For instance, the honeycomb lattice (HCL $)^{33}$ is composed of two triangular sublattices $(A, B)$, which features conical intersections with two touching bands at two inequivalent Dirac points $\left(K\right.$ and $\left.K^{\prime}\right)$, representing a half-integer pseudospin system $(S=$ $1 / 2$, see Fig. 1a). In contradistinction, the Lieb lattice ${ }^{34-36}$ has three square sublattices $(A, B, C)$, which possess a conical intersection with three touching bands at the Dirac-like $M$ points, representing an integer pseudospin system $(S=1$, see Fig. 1b).

Crucially, since the pseudospin operators satisfy a set of commutation relations directly analogous to those used to define the real spin, pseudospin should be treated on equivalent footing as other angular momenta in a given system. Consequently, a whole class of fundamental phenomena based on pseudospin-orbit interaction, twined together with topology of the underlying space, should be expected in photonic, electronic, and other relevant platforms with emergent pseudospins. We demonstrate here one such phenomenon using a photonic platform: momentum-to-realspace mapping of topological singularities.

In the paraxial approximation, light propagation in photonic lattices is governed by the Schrödinger equation ${ }^{13,33}$ :

$$
\mathrm{i} \frac{\partial \Psi(x, y, z)}{\partial z}=-\frac{1}{2 k_{0}} \nabla^{2} \Psi(x, y, z)-\frac{k_{0} \Delta n(x, y)}{n_{0}} \Psi(x, y, z) \equiv H_{0} \Psi
$$

where $\Psi$ is the optical field of the probe beam, $z$ is the longitudinal propagation distance, $k_{0}$ is the wavenumber, $n_{0}$ is the background refractive index of the medium, and $\Delta n$ is the induced index change forming either the HCL or the Lieb lattice. In Eq. (1), $H_{0}$ is the continuous Hamiltonian of the system, whose eigenvalues define the lattice band structure. For the HCL, Eq. (1) becomes a a

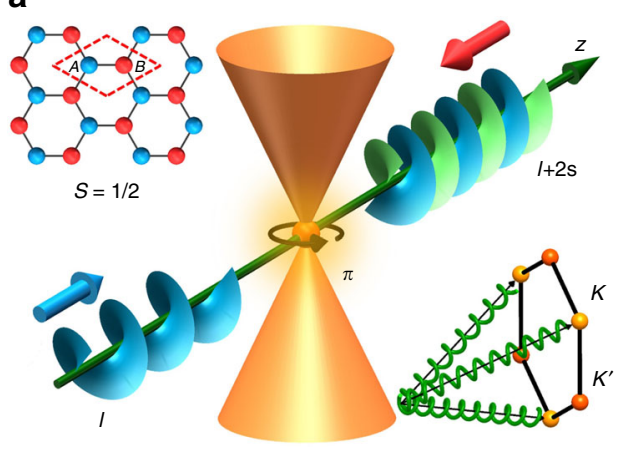

b

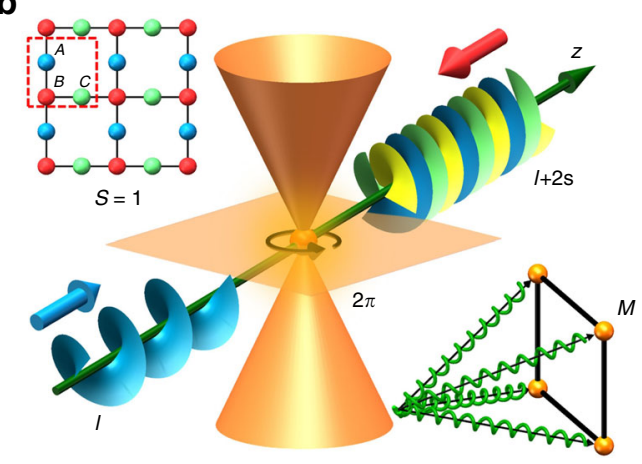

C

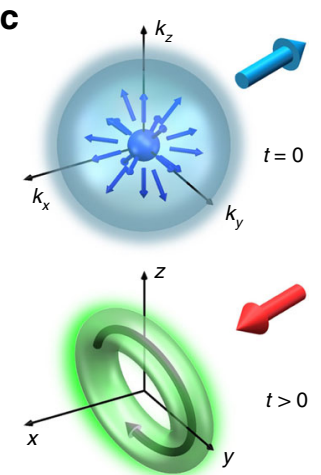

Fig. 1 Illustration of momentum-to-real-space mapping of topological singularities. a $A$ pseudospin- $1 / 2$ honeycomb lattice with two sublattices $A$ and $B$ is excited with three vortex beams, each with topological charge I. b A pseudospin- 1 Lieb lattice with three sites ( $A, B, C$ ) per unit cell is excited with four vortex beams. These vortex beams excite modes around conical intersections at the corners of the Brillouin zone (lower right inset). The arrows circulating around the conical intersections illustrate winding of the Berry phase ( $\pi$ in $\mathrm{HCL}$ and $2 \pi$ in Lieb lattice). Topological charge conversion from $/$ to $I+2 s$ is a consequence of the mapping of topological singularity from momentum to real space. It occurs when initial excitation with $I= \pm 1$ is optimally aligned for a given pseudospin state (a) $s= \pm 1 / 2$ in $\mathrm{HCL}$, and (b) $s= \pm 1$ in Lieb lattice. c Illustration of similar excitation of a Berry curvature monopole in 3D momentum space, leading to generation of a topological charge in real space with vorticity along the direction of excitation. Small blue and red arrows depict opposite pseudospin states. 

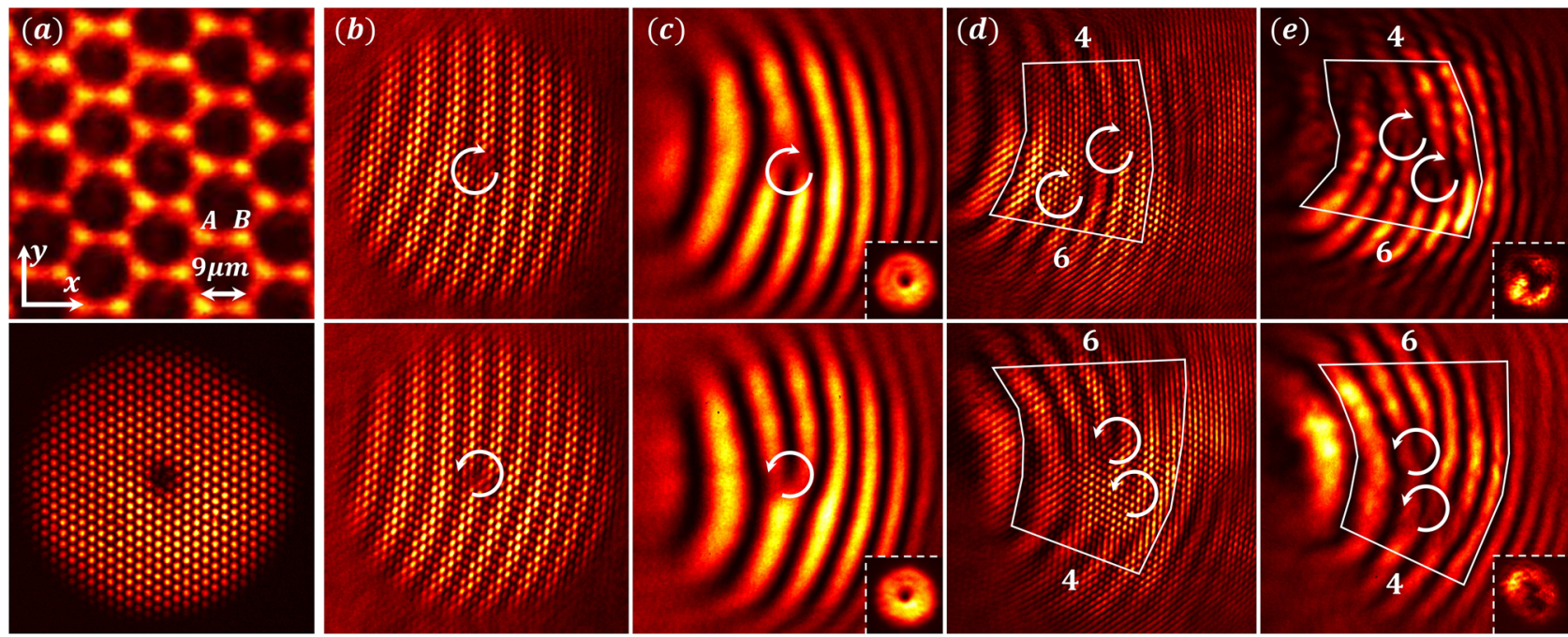

Fig. 2 Experimental demonstration of topological conversion in a pseudospin-1/2 honeycomb lattice. a Top: an optically induced $H C L$; Bottom: input pattern of vortex-bearing triangular lattice beam used for selective excitation of the pseudospin states. b-e Top (bottom) row corresponds to initial excitation of $s=1 / 2(s=-1 / 2)$ pseudospin state with vortex beams of initial topological charge $I=1(I=-1)$. Interferograms of input (b, $c)$ and output (d, e) with a tilted reference beam showing topological charge conversion from 1 to 2 (top) and from -1 to -2 (bottom). $\mathbf{b}$, $\mathbf{d}$ Interferogram from the whole beam, and $(\mathbf{c}, \mathbf{e})$ corresponding interferogram from one of the spectral components. Difference in the numbers of counted fringes from the two sides of the marked region illustrates the net topological charges at output in (d, e). White curved arrows mark the position and helicity of the vortices. Insets in (c) and (e) show singly and doubly-charged vortex intensity patterns obtained at input and output, respectively, from one of the $K$ valleys as illustrated in Fig. 1 a.

two-band simplified description of the paraxial model under the tight-binding approximation, and for excitations near the Dirac points, it turns into the linear Dirac equation typically used for describing massless Dirac particles in graphene ${ }^{37}$. The amplitude of the optical wave in the two sublattices is then modeled by twocomponent spinor functions corresponding to pseudospin. In our previous work ${ }^{13}$, the angular momentum associated with such lattice pseudospin has been observed. However, the topological properties arising from the interplay between pseudospin-orbit interaction and nontrivial Berry phases remain largely unexplored.

\section{Results}

Topological conversion in a pseudospin-1/2 honeycomb lattice. For excitations around conical intersections in both lattices, the dynamics is governed by the effective Hamiltonian

$$
H=\kappa\left(S_{x} k_{x}+S_{y} k_{y}\right)
$$

where $S_{i}$ are the components of the pseudospin angular momentum operator $\mathbf{S}, k_{x}$ and $k_{y}$ are the displacements of the transverse wavevectors with respect to the Dirac point, and $\kappa$ depends on the properties of the lattice. The eigenstates of the pseudospin $\chi_{S, s}$ are given by $\mathbf{S}^{2} \chi_{S, s}=S(S+1) \chi_{S, s}$, and $S_{z} \chi_{S, s}=s \chi_{S, s}$ (here $S$ and $s$ denote the total and $z$-component of the pseudospin angular momentum, respectively). The eigenmodes of the Hamiltonian, $H \psi_{n, k}=\beta_{n, k} \psi_{n}$, $k$, are organized in $2 S+1$ bands (labeled by $n$ ) touching at the conical intersection. In contrast to previous excitation schemes, we use three vortex beams (each with an initial topological charge $l=$ 1 or $l=-1)$ momentum-matched to the conical intersection points for the HCL (see Fig. 1a), spatially structured to excite only one pseudospin eigenstate $(s=-1 / 2$ or $1 / 2)$. Likewise, for the Lieb lattice, we use four vortex beams (see Fig. 1b) to excite one pseudospin eigenstate $(s=-1,0$ or 1$)$ for each measurement. Further experimental details about lattice creation in a $20-\mathrm{mm}$ long nonlinear crystal (SBN:61) and excitation scheme can be found in Supplementary Note 1.

Typical experimental results obtained with the HCL are summarized in Fig. 2. The HCL (Fig. 2a) is established with the multi-beam optical induction technique ${ }^{38,39}$. It remains invariant throughout the crystal with a nearest neighbor spacing of $9 \mu \mathrm{m}$. The lattice is probed by a donut-shaped triangular lattice beam, for which the OAM $(l)$ and pseudospin $(s)$ are optimally aligned (top panel: $l=1, s=1 / 2$, bottom panel $l=-1, s=-1 / 2$ ). To better see the phase structure of the probe beam at input (i.e., before the pseudospin-orbit interaction takes place), interferograms are obtained for the whole superimposed beam (Fig. 2b) as well as one of the three interfering beams (Fig. 2c). As illustrated in Fig. 1a, the vortex beams are momentum-matched to the three equivalent Dirac points $(K)$ of the HCL. The output interferograms in Fig. 2d, e clearly display two vortices of the same helicity, which show conversion of the topological charges from $l$ to $l+2$ s for both initial states (schematically illustrated in Fig. 1a). We demonstrate below that this conversion is a consequence of the mapping of topological singularity at the conical intersections from momentum to real space. Figure $2 \mathrm{e}$ is obtained from the Fourier transform of spectral component at one of the Dirac points back into real space for phase measurement. The bottom-right inset in Fig. 2e shows a donutshaped intensity pattern at the output, which is somewhat deformed as compared to the input (see the inset in Fig. 2c) because it is now a higher-order vortex which tends to disintegrate into multiple singly-charged vortices during propagation in an inhomogeneous medium ${ }^{40}$.

It is instructive to provide a kinematical explanation of these observations (see Supplementary Note 2). In the experiment of Fig. 2, the $z$-component $J_{z}$ of the total angular momentum $\mathbf{J}=$ $\mathbf{L}+\mathbf{S}$ is conserved (where $\mathbf{L}=\mathbf{r} \times \mathbf{k}$ is the OAM): $\left[J_{z}, H\right]=0$. The initial excitation in all experiments is comprised of a single value of $l$ and $s$, and the optimally aligned initial condition implies maximal value of $|l+s|$. The output beam has two or more values of $l^{\prime}$ and $s^{\prime}$, all of which obey

$$
l+s=l^{\prime}+s^{\prime} .
$$

For example, for Fig. 2-top panel, the input beam has $l=1$ and $s=1 / 2$, while the output beam has two components: (i) $l^{\prime}=1$ and $s^{\prime}=1 / 2$, and (ii) $l^{\prime}=2$ and $s^{\prime}=-1 / 2$. Since the output 

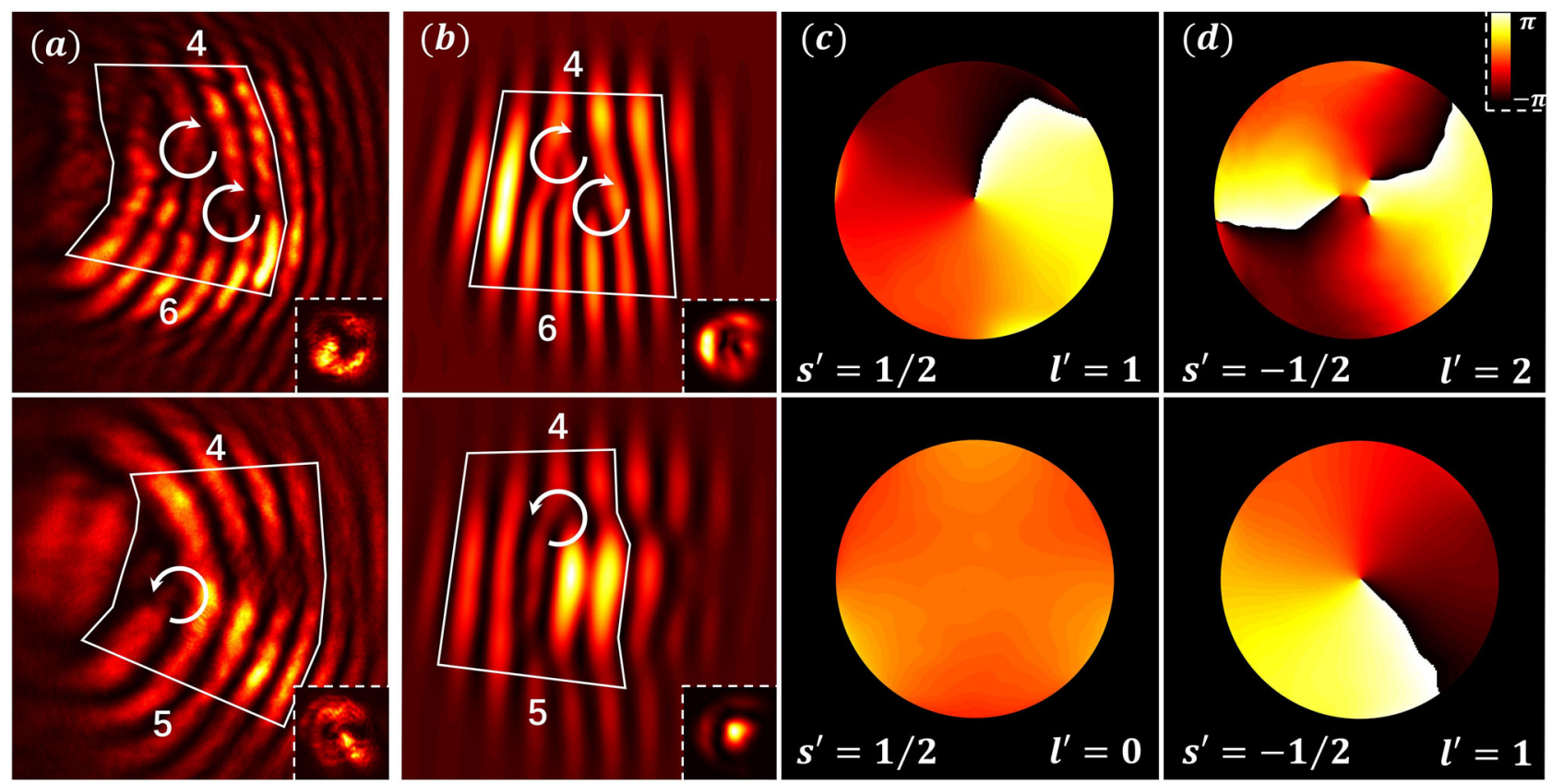

Fig. 3 Decomposed pseudospin components in an angular-momentum-conserved HCL. Top (bottom) row: pseudospin state $s=1 / 2$ ( $s=-1 / 2$ ) is selectively excited with initial beam of topological charge $I=1$. a Output interferogram from the experiment, and (b) corresponding results from the simulation with $(\mathbf{c}, \mathbf{d})$ showing the evolved phase structure separately for each pseudospin component. The topological charge increases (decreases) by 1 unit in the initially unexcited component as seen in the top (bottom) panel of $\mathbf{d}$ (c), while that in the initially excited component remains intact, as governed by Eq. (3). The lower insets in $(\mathbf{a}, \mathbf{b})$ are the corresponding intensity patterns; the doughnut (central bright spot) pattern corresponds to $I \neq 0$ ( $=0$ ) components.

components are intertwined on both sublattices, we observe two vortices in Fig. 2d, e. A fully equivalent explanation holds for results in Fig. 2-bottom panel. However, as we shall discuss below through theoretical analysis, the observed charge conversion has a topological origin, holding even in systems without rotational symmetry in which angular momentum is not conserved.

To substantiate the above kinematical picture summarized in Eq. (3), experimental observations (Fig. 3a) are further corroborated by numerical simulations (Fig. $3 \mathrm{~b}-\mathrm{d}$ ) based on the paraxial wave equation (Eq. (1)). For all simulations, the parameters are chosen close to those from experiment with the index contrast $\delta n=2 \times 10^{-4}$.We excite the pseudospin states $s=1 / 2$ (top) and $s=-1 / 2$ (bottom), with the input beam of topological charge $l=$ 1 covering sublattices $A$ and $B$, respectively. In numerical simulations, the output field is decomposed into each pseudospin component. From the phase structure of each component, the difference is clear: if the $s=1 / 2$ component is initially excited, the unexcited $s^{\prime}=-1 / 2$ component is converted into an $l^{\prime}=2$ vortex (Fig. 3d, top). In contrast, if the $s=-1 / 2$ is initially excited, the vorticity in the unexcited $s^{\prime}=1 / 2$ component disappears, $l^{\prime}=0$ (Fig. 3c, bottom). The vorticity of the initially excited component always remains unchanged, in accordance with Eq. (3). Note that the output intensity patterns in the lower insets of Fig. 3 have a subtle difference between the two cases of excitation: the donut shape is preserved in the top panels (when both components maintain a vortex), but deforms to have a bright central spot in bottom panels (when vortex annihilation occurs in one of the components).

Topological conversion in a pseudospin-1 Lieb lattice. Next, we discuss experimental results obtained with a photonic Lieb lattice (summarized in Fig. 4). The lattice is established again by optical induction in the 20 -mm-long crystal ${ }^{36}$, with a nearest neighbor spacing of $9 \mu \mathrm{m}$. A donut-shaped square lattice beam (Fig. 4a, bottom) created by interfering four singly-charged vortex beams is employed as a probe, which excites only one pseudospin component (top panel: $l=1, s=1$; bottom panel: $l=-1, s=-1$ ). In the Lieb lattice, the pseudospin $S_{z}$ is not diagonal in sublattice basis ${ }^{15}$. Therefore, to excite a given pseudospin state, the probing square lattice is matched either to the $B$ sublattice (for the $s=0$ pseudospin state) or the $A$ and $C$ sublattices with appropriate phase relation (for the $s=1$ and $s=-1$ pseudospin states) as illustrated in Fig. 4b. The output interferograms in Fig. 4e clearly display three vortices of the same helicity as the input (i.e., a net topological charge of 3 or -3 ), exhibiting a conversion of the topological charges from $l$ to $l+2 \mathrm{~s}$ for the optimally aligned excitations (schematically illustrated in Fig. 1b).

In Fig. 5, we show experimental (Fig. 5a) and numerical (Fig. 5b) results obtained by initial excitation of the pseudospin states (from top to bottom rows) $s=1, s=0$, and $s=-1$ with a proper input beam of topological charge $l=1$, and examine how the phase evolves for the three decomposed pseudospin components (Fig. $5 \mathrm{c}-\mathrm{e}$ ). The first case $(s=1)$ corresponds to optimally aligned excitation in Fig. 4, where the topological charge emerging in the $s^{\prime}=-1$ pseudospin component is $l^{\prime}=3$. For the latter two cases $(s=0$, and $s=-1)$, which are not optimally aligned, the initial vortex is also transformed into multiple vortices but with a net topological charge of 2 (middle row) or 1 (bottom row), while all components satisfy again the kinematics of pseudospin-orbit interaction (Eq. (3)). Similar studies with the input beam of topological charge $l=-1$ led to the same conversion rule. The pseudospin components of the Lieb lattice are not diagonal in the sublattice basis, and therefore do not have a trivial correspondence to a particular sublattice ${ }^{15}$ as for the case of pseudospin-1/2 $\mathrm{HCL}^{13}$. In fact, the physics of pseudospin-orbit interaction in Lieb lattices is in one aspect richer than that of polarization-based spin-orbit interaction: here we have excited also the $s=0$ pseudospin state, inadmissible for helicity of photons due to its zero mass. 

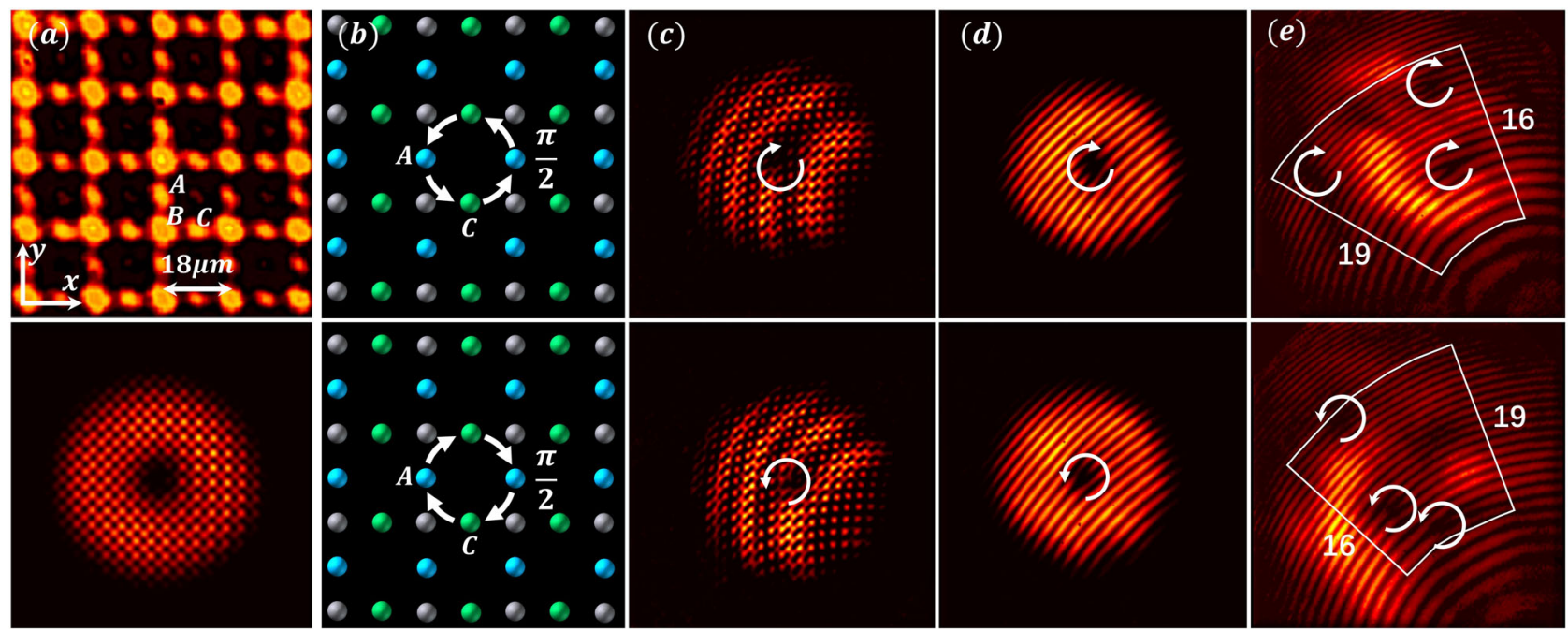

Fig. 4 Experimental demonstration of topological conversion in a pseudospin-1 Lieb lattice. a Top: an optically induced Lieb lattice; Bottom: input pattern of vortex-bearing square lattice beam used to excite the pseudospin states of the Lieb lattice. $\mathbf{b}$ Top (bottom) row illustrates selective excitation of $A$ and $C$ sublattices with an appropriate phase relation optimized for pseudospin states $s=1(s=-1)$ by vortex beams of initial topological charge $I=1(I=-1)$. c-e Interferograms of input (c, d) and output (e) showing topological charge conversion from 1 to 3 (top) and from -1 to -3 (bottom).
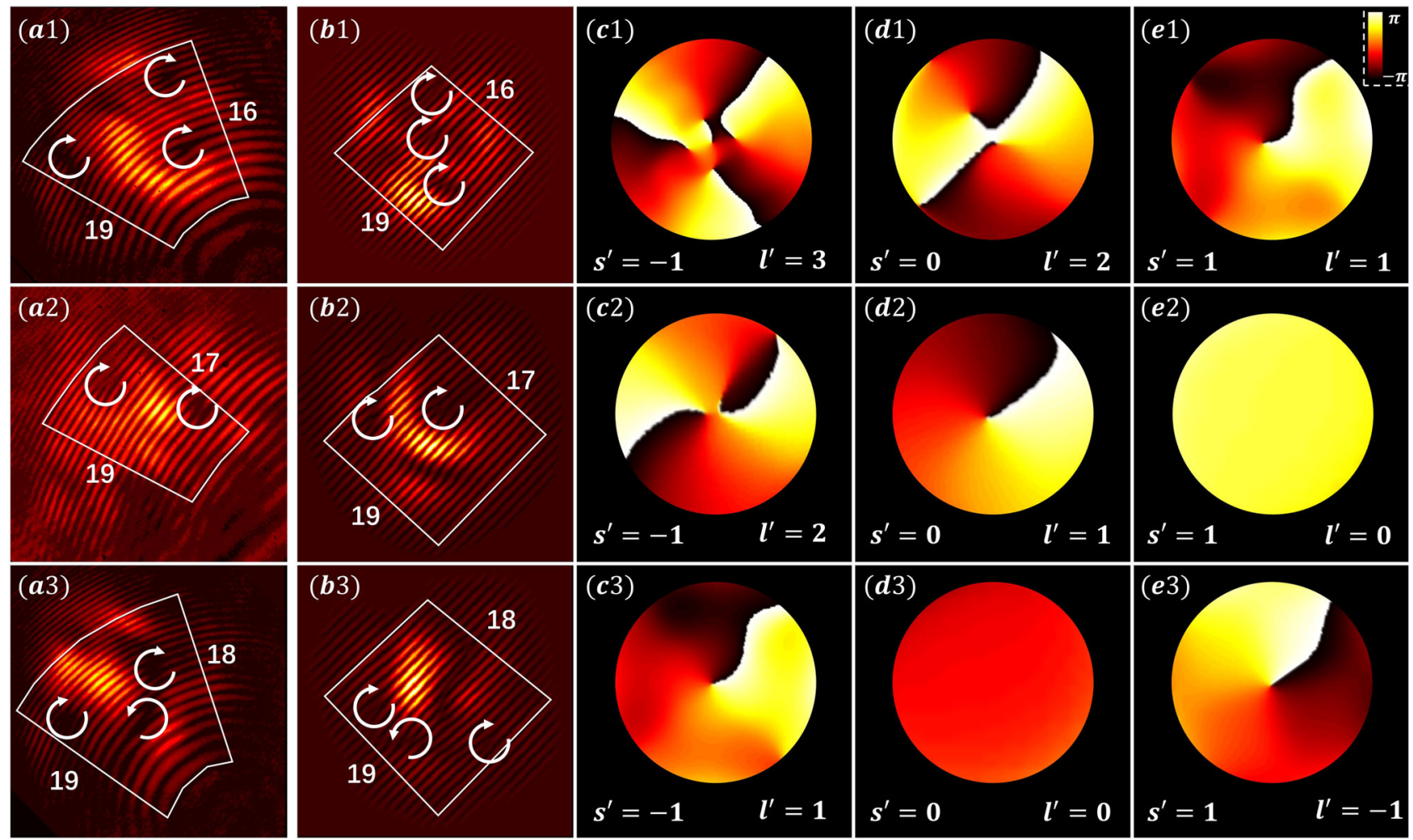

Fig. 5 Decomposed pseudospin components in an angular-momentum-conserved Lieb lattice. Experiment and simluation results for initial excitations of the pseudospin states $s=0$ in (a1-e1), $s=1$ in (a2-e2) and $s=-1$ in (a3-e3) with four input beams of topological charge $I=1$. Output interfrograms from (a) experiment and (b) simulation show different topological charge conversions under different excitation conditions. (c-e) show output phase structure of the probe beam numerically decomposed for each pseudospin component $s^{\prime}$, where corresponding output vorticity $I^{\prime}$ in each component has been identified. In all cases, each pseudospin component obeys Eq. (3).

Fundamental mechanism of the topological charge conversion. Up to this point, we have discussed topological charge conversion in honeycomb and Lieb lattices and explained the results kinematically with angular momentum conservation. One can also explain the observed phenomenon dynamically, by expanding the initial excitation in eigenmodes of the Hamiltonian, as elaborated in the Methods section. However, there is a fundamental topological mechanism beyond the charge conversion observed in experiment. When eigenmodes of the Hamiltonian (2) are expanded in pseudospin eigenstates, there are vortices (i.e., topological charges) in the $k$-space attached to the components, and the topological charge of neighboring pseudospin components 
differs by one. For example, for the HCL the eigenmodes are $\psi_{n, \mathbf{k}}=\frac{1}{\sqrt{2}}\left(\begin{array}{c}n \\ \mathrm{e}^{\mathrm{i} \varphi_{k}}\end{array}\right)=\frac{1}{\sqrt{2}}\left(n \chi_{\frac{1}{2}, \frac{1}{2}}+\mathrm{e}^{\mathrm{i} \varphi_{k}} \chi_{\frac{1}{2},-\frac{1}{2}}\right)$ (see Methods and Supplementary Notes). When a single pseudospin component is excited, the $k$-space vortex in the other component is mapped from momentum to real space, giving rise to topological charge conversion. Difference in the $k$-space topological charges of pseudospin components is related to the Berry phase winding around the Dirac point (see Methods). If we denote the winding of the Berry phase around the Dirac point with $w \pi$, then for the HCL, $w=1$; and for the Lieb lattice, $w=2$. Topological quantity $w$ is also the maximal difference between the $k$-space topological charges of pseudospin components. For the studied honeycomb and the Lieb lattices, the rule $l \rightarrow l+2 s$, which holds only for optimally aligned excitations, can be expressed as $l \rightarrow l+w$ for $l>$ 0 (or $l \rightarrow l-w$ for $l<0$ ). It turns out that this latter expression of the conversion rule, which contains the topological quantity $w$, is more general than the one containing pseudospin $s$, as can be viewed on the following examples.

Consider a conical intersection described by the Hamiltonian $H_{s}=\kappa_{x} S_{x} k_{x}+\kappa_{y} S_{y} k_{y}$, where the angular momentum is not conserved for $\kappa_{x} \neq \kappa_{y}$ due to lack of rotational symmetry; an inspection of the eigenstates of $H_{s}$ for pseudospin $S=1 / 2$ and $S=1$ shows that the $k$-space vortices become elliptical but preserve their topological charge. For the stretched HCL, the winding of the Berry phase around the Dirac point is protected, until the stretching is sufficiently large so that the inequivalent Dirac points merge and a gap opens ${ }^{41-43}$. After this transition the Berry phase vanishes and the topological charge conversion no longer occurs. In Fig. 6 we show numerical simulations for the optimally aligned initial condition in the stretched HCL and Lieb lattice. The nearest neighbor spacing for the HCL and the Lieb lattice is $9 \mu \mathrm{m}$, stretched by $12 \%$ and $15 \%$, respectively. As seen from these results, the conversion of the topological charges from $l$ to $l \pm w$ holds even when the angular momentum $J_{z}$ is not conserved, which indicates that the mapping of the topological charges from $k$-space to $x$-space is a fundamental process with topological origin. Other examples include band structures with parabolic band-touching points, or conical intersections described by the Hamiltonian $H_{0}=k \sigma_{z}$, where the formula $l \rightarrow l \pm w$ still holds, further underpinning the topological interpretation of our results (see Methods and Supplementary Notes).

Next, we expand our results beyond the 2D platform. Our experiments are performed in 2D lattices; however, analogous considerations can be made for 3D Hamiltonians. For example, we consider the Weyl Hamiltonian $H_{\mathrm{Weyl}}=\boldsymbol{\sigma} \cdot \mathbf{k}$, which has attracted considerable interest in recent years; it has been experimentally realized in the Brillouin zone of specially designed optical and condensed matter structures ${ }^{16,17}$. This Hamiltonian gives rise to a synthetic magnetic monopole in $k$-space ${ }^{18}$, with topological charge 1 . Suppose that we initially excite the modes around a Weyl point with a symmetric Gaussian-like distribution, and that we excite a pseudospin eigenstate $\chi_{\frac{1}{2}, \frac{1}{2}}$. The initial state is $\psi_{0, \frac{1}{2}}\left(r, \theta_{r}, \varphi_{r}, t=0\right)=\psi_{0}(r) \chi_{\frac{1}{2}, \frac{1}{2}}$, and its evolution in time is governed by $\mathrm{i} \frac{\partial \psi}{\partial t}=H_{\mathrm{Weyl}} \psi$. By expanding the initial state in eigenmodes of the Weyl Hamiltonian, it is straightforward to see that the wavefunction evolves as (see Supplementary Notes for details)

$$
\psi_{0, \frac{1}{2}}\left(r, \theta_{r}, \varphi_{r}, t\right)=\chi_{\frac{1}{2}, \frac{1}{2}} g_{\frac{1}{2}, \frac{1}{2}}\left(r, \theta_{r}, t\right)+\mathrm{e}^{\mathrm{i} \varphi_{r}} \chi_{\frac{1}{2},-\frac{1}{2}} g_{\frac{1}{2},-\frac{1}{2}}\left(r, \theta_{r}, t\right) .
$$

Clearly, even though the initial state was a Gaussian-like excitation with $l=0$ and $s=1 / 2$, in the unexcited pseudospin component $s^{\prime}=-1 / 2$, a vortex with topological charge $l^{\prime}=1$ emerges, as illustrated in Fig. 1c. Since this is a 3D rotationally invariant Hamiltonian, $\left[\mathbf{J}, H_{\text {Weyl }}\right]=0$, if we excite any pseudospin state $\alpha \chi_{\frac{1}{2}, \frac{1}{2}}+\beta \chi_{\frac{1}{2},-\frac{1}{2}}$ with a Gaussian-like distribution, we will obtain in the output a vortex field with a topological charge identical to the charge of the Weyl monopole, with vorticity pointing in the direction of the initial pseudospin. Thus, with properly designed initial excitation, mapping of topological properties of the Weyl
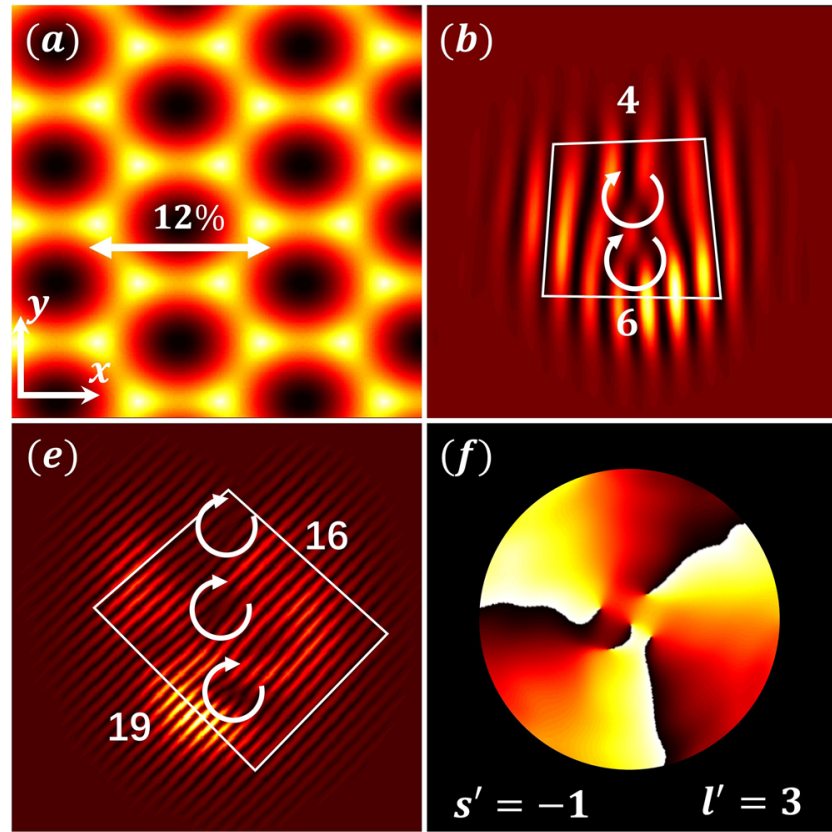

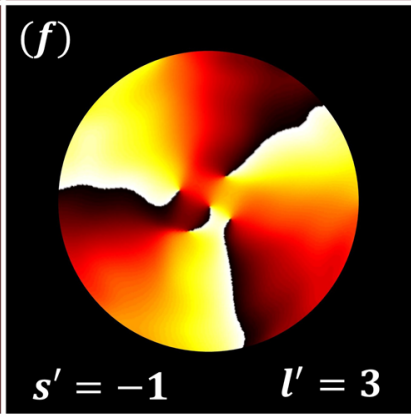

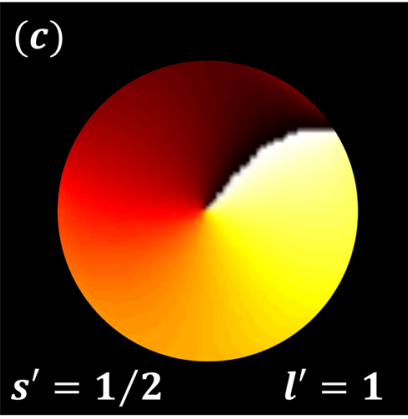
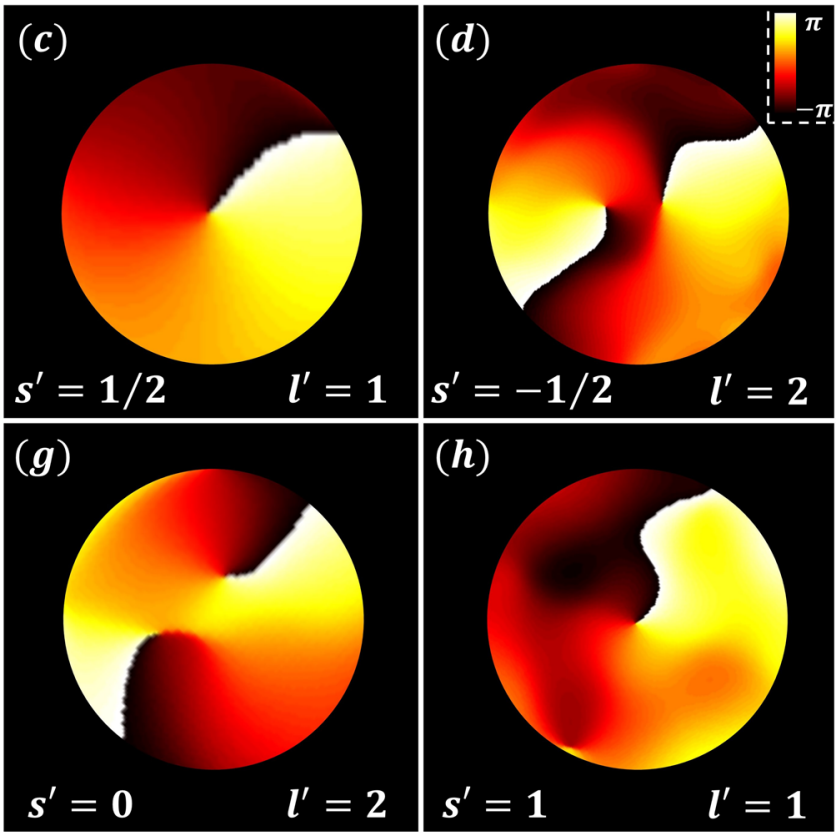

Fig. 6 Evolution of pseudospin states in stretched lattices lacking rotational symmetry around conical intersections. Top (bottom) row corresponds to a stretched HCL (Lieb lattice), with an initial excitation $I=1, s=1 / 2(I=1, s=1)$. a A $12 \%$ horizontally stretched HCL. $\mathbf{b}$, e Interferograms of the output beam, which clearly indicate the topological conversion from $I$ to $I+2 s$ (or $I \pm w$ ), corresponding to results from unstretched lattices of the top rows in Figs. $3 b$ and $5 \mathrm{~b}$. This is underpinned by the phase structure of the pseudospin components at the output illustrated in (c, d) and (f-h) for the two lattices. 
monopole to topological charges in real space can be readily achieved. In fact, this type of dynamics in 3D Weyl systems, achievable in ultracold atomic gases ${ }^{19}$, seems to be related to a recent experiment where an electron beam scattered from a magnetic monopole experienced a conversion into an electron vortex ${ }^{20}$.

We have thus demonstrated the universal mapping of topological singularities in $k$-space to measurable topological entities in real space. Our experiments were carried out in photonic honeycomb and Lieb lattices, where the mapping can be explained with pseudospin-orbit interaction, angular momentum conservation and nontrivial winding of the Berry phase. However, we have demonstrated in theory that the underlying mechanism for the mapping lies in fundamentally topological origin. Besides the typical honeycomb and Lieb conical intersections, we have shown that it occurs also in stretched lattices where angular momentum is not conserved, and for parabolic band touching and other nonconical intersections. Moreover, we have predicted that the same mechanism exists in 3D Weyl lattices where synthetic magnetic monopoles come to play the role. Our finding brings about many interesting questions as well as opportunities. For instance, is it possible to create vortices of Bose-Einstein condensates $^{44}$ by topological conversion from synthetic magnetic monopoles in ultracold atomic gases ${ }^{19}$ ? How could the mechanism explored here be adapted for topological conversion with photons in a photonic Dirac monopole field ${ }^{45}$ ? It is also natural to ask: is the spin angular momentum gifted by light polarization indispensable in spin-to-orbital angular momentum conversion, as commonly thought, or the pseudospin and topological conversion is essential even in those conventional settings based on optical phase elements ${ }^{24,26}$ ? What other mechanisms can we conceive and explore where topological properties of the bands can be directly mapped from momentum to real space in experiments? Can other topological entities such as vortex knots and nodal chains ${ }^{46-48}$ be directly mapped from momentum space to real space or vice versa, or onto a synthetic space ${ }^{49,50}$ ?

\section{Methods}

Theoretical framework for topological conversion. We develop here a systematic theoretical framework to fully analyze the observed phenomena, which unravels the connections between pseudospin, OAM and the underlying topology of the lattice in $k$-space. The initial vortex beam which probes the conical intersection of Hamiltonian in Eq. (2) is described with the complex amplitude of the electric field $\psi_{l, s}\left(r, \varphi_{r}, z=0\right)=\psi_{0} r^{l} \mathrm{e}^{\mathrm{i} l \varphi_{r}} \exp \left(-r^{2} / a_{0}^{2}\right) \chi_{S, s}$, where $\chi_{S, s}$ accounts for the fact that we initially excite only a single pseudospin component. The probe beam is broad in real space $\left(a_{0} \gg\right.$ lattice constant), and narrow in momentum space. We expand the initial excitation in eigenmodes of the Hamiltonian (2) and account for the

dynamics via $\psi_{l, s}\left(r, \varphi_{r}, z\right)=\sum_{n, \mathbf{k}} c_{n, \mathbf{k}} \psi_{n, \mathbf{k}} \exp \left(-\mathrm{i} \beta_{n, \mathbf{k}} z\right)$; the coefficients $c_{n, k}$ are found by calculating projection $\left\langle\psi_{n, k} \mid \psi_{l, s}(z=0)\right\rangle$ (see Supplementary Notes). For the optimally aligned initial state in HCL (e.g., exciting the $s=1 / 2$ state with $l=1$ ), it is straightforward to find the evolving complex amplitude of the electric field:

$$
\psi_{l=1, s=\frac{1}{2}}\left(r, \varphi_{r}, z\right)=\mathrm{e}^{\mathrm{i} \mid \varphi_{r}} \chi_{\frac{1}{2}, \frac{1}{2}} g_{\frac{1}{2}, \frac{1}{2}}(r, z)+\mathrm{e}^{\mathrm{i}(l+1) \varphi_{r}} \chi_{\frac{1}{2},-\frac{1}{2}} g_{\frac{1}{2},-\frac{1}{2}}(r, z) .
$$

The radial and $z$-dependence of the electric field is for clarity denoted with $g_{\frac{1}{2}, \pm \frac{1}{2}}(r, z)$, and it produces the conical diffraction pattern ${ }^{13,33}$. This is in full agreement with observations in Figs. 2 and 3 (top rows), which show that the initially unexcited $s^{\prime}=-1 / 2$ component has vorticity $l^{\prime}=l+1$, whereas the excited $s^{\prime}=1 / 2$ component has vorticity $l^{\prime}=l$.

In a fully equivalent manner, we can describe the dynamics in the Lieb lattices. For the optimally aligned initial excitation (e.g., exciting the $s=1$ state with $l=1$ ), the electric field evolves according to the following (see Supplementary Notes):

$\psi_{l=1, s=1}\left(r, \varphi_{r}, z\right)=\mathrm{e}^{\mathrm{i} l \varphi_{r}} \chi_{1,1} g_{1,1}(r, z)+\mathrm{e}^{\mathrm{i}(l+1) \varphi_{r}} \chi_{1,0} g_{1,0}(r, z)+\mathrm{e}^{\mathrm{i}(l+2) \varphi_{r}} \chi_{1,-1} g_{1,-1}(r, z)$

We see that the topological charge emerged in the pseudospin components $s=0$ and -1 is $l+1$ and $l+2$, respectively, in accordance with experimental results and numerical simulations presented in Figs. 4 and 5 (top rows), and the kinematical arguments. Dynamical considerations provide, in addition, the details of the $r$ - and $z$-dependence of the electric field, which are contained in the gfunctions. For all other initial conditions, similar calculations yield results also in accordance with observations.
Although the above dynamical explanation seems enough to address the observed phenomena, there is a fundamental connection to the underlying topology of $k$-space and the observation of vortices in $x$-space. If a beam propagates sufficiently long in a photonic lattice, as in our experiments, the output intensity of the beam in $x$-space will reflect the initial distribution of the power in the lattice $k$-space (analogous to farfield dynamics in free space). However, the HCL possesses topological singularity at the Dirac point: the Berry phase acquired as one traverses a loop around the Dirac point is $\pi$; the Dirac point can be considered as a flux tube (topological singularity) of the Berry curvature ${ }^{51}$. Our experiments essentially reveal how the excitations of modes around the singularity are mapped into the far field dynamics.

To see that clearly, we revisit the calculation of the Berry phase in the HCL. An HCL eigenstate close to the conical intersection can be written as $\psi_{n, \mathbf{k}}=\frac{1}{\sqrt{2}}\left(\begin{array}{c}n \\ \mathrm{e}^{\mathrm{i} \varphi_{k}}\end{array}\right)$, with $n= \pm 1$. As we adiabatically circle around the Dirac point, the acquired Berry phase is $-\mathrm{i} \oint\left\langle\psi_{n, \mathbf{k}}\left|\frac{\partial}{\partial \varphi_{k}}\right| \psi_{n, \mathbf{k}}\right\rangle \mathrm{d} \varphi_{k}=\pi$. The Berry phase arises from the specific phase relation in $k$-space between the pseudospin components of the eigenstate. There is a vortex (i.e., a topological charge) in $k$-space in one of the pseudospin components; more precisely, the difference in $k$-space topological charges of the two components is one. From the derivation of Eq. (5), we see that during propagation this vortex is mapped from the $k$-space to the $x$-space (see Supplementary Notes). Thus, what we observed in our experiments is the topological singularity of the HCL mapped from momentum to real space. The mapping is revealed due to the properly designed initial excitation of a single pseudospin state.

This finding holds for a general Hamiltonian (2), i. e., for any pseudospin $S$. Every eigenmode can be expanded in pseudospin eigenstates as

$\psi_{n, \mathbf{k}}=\sum_{s=-S}^{S}\left\langle\chi_{S, s} \mid \psi_{n, \mathbf{k}}\right\rangle \chi_{S, s}$. The coefficients $\left\langle\chi_{S, s} \mid \psi_{n, \mathbf{k}}\right\rangle$ are found by rewriting the Hamiltonian as $H=\frac{\kappa}{2} k\left(S_{+} \mathrm{e}^{-\mathrm{i} \varphi_{k}}+S_{-} \mathrm{e}^{\mathrm{i} \varphi_{k}}\right)$, where $S_{ \pm}=S_{x} \pm \mathrm{i} S_{y}$ :

$$
\begin{aligned}
\beta_{n, \mathbf{k}}\left\langle\chi_{S, s} \mid \psi_{n, \mathbf{k}}\right\rangle= & \left\langle\chi_{S, s}|H| \psi_{n, \mathbf{k}}\right\rangle \\
= & \frac{\kappa}{2} k\left(\sqrt{(S-s)(S+s+1)}\left\langle\chi_{S, s+1} \mid \psi_{n, \mathbf{k}}\right\rangle \mathrm{e}^{-\mathrm{i} \varphi_{k}}\right. \\
& \left.+\sqrt{(S+s)(S-s+1)}\left\langle\chi_{S, s-1} \mid \psi_{n, \mathbf{k}}\right\rangle \mathrm{e}^{\mathrm{i} \varphi_{k}}\right) .
\end{aligned}
$$

There is a clear phase relationship between different pseudospin components of the eigenstates. The difference in $k$-space topological charges (vortices) of neighboring pseudospin components is one. When a single pseudospin component is excited, $k$-space topological charges of the unexcited components are mapped to real space, which is the fundamental mechanism behind topological charge conversions observed in our experiments.

To further corroborate the topological interpretation of the conversion, let us consider the following two examples. The first is the Hamiltonian

$$
H_{m}=\left(\begin{array}{cc}
0 & \left(k_{x}-\mathrm{i} k_{y}\right)^{m} \\
\left(k_{x}+\mathrm{i} k_{y}\right)^{m} & 0
\end{array}\right),
$$

which for $m=1$ corresponds to the HCL we studied above. The band structure for $m=2$ corresponds to bilayer graphene and has a parabolic band-touching point, and for $m>2$ it corresponds to other variants of band touching. It is straightforward to verify that if the nonconical Dirac-like points are excited with a fully equivalent optimal excitation as for the HCL, the topological charge conversion $l \rightarrow l \pm w$ still holds. However, the expression $l \rightarrow l+2 s$ relying on the angular momentum is no longer applicable. Note that for the Lieb and bilayer graphene lattices, the topological conversion arises from the Berry phase of $2 \pi$, which may seem trivial at glance ${ }^{52}$. In a similar fashion, one may explore another example of the Hamiltonian $H_{0}=k \sigma_{z}$, which has a conical intersection point like the HCL case but here with $w=0$. It can be shown again that $l \rightarrow l \pm w$ is valid, consolidating our finding that a more general interpretation of our experimental observations should be formulated by the winding of the Berry phase as the topological quantity, rather than just the pseudospin.

\section{Data availability}

The data that support the findings of this study are available from the corresponding author upon reasonable request.

Received: 18 September 2019; Accepted: 4 March 2020;

Published online: 27 March 2020

\section{References}

1. Klitzing, K. V., Dorda, G. \& Pepper, M. New method for high-accuracy determination of the fine-structure constant based on quantized Hal resistance. Phys. Rev. Lett. 45, 494-497 (1980).

2. Kane, C. L. \& Mele, E. J. Quantum spin Hall effect in graphene. Phys. Rev. Lett. 95, 226801 (2005)

3. Lu, L., Joannopoulos, J. D. \& Soljačić, M. Topological photonics. Nat. Photon 8, 821-829 (2014). 
4. Ren, Y., Qiao, Z. \& Niu, Q. Topological phases in two-dimensional materials: a review. Rep. Prog. Phys. 79, 066501 (2016).

5. Ozawa, T. et al. Topological photonics. Rev. Mod. Phys. 91, 015006 (2019).

6. Ma, G., Xiao, M. \& Chan, C. T. Topological phases in acoustic and mechanical systems. Nat. Rev. Phys. 1, 281-294 (2019).

7. Cooper, N. R., Dalibard, J. \& Spielman, I. B. Topological bands for ultracold atoms. Rev. Mod. Phys. 91, 015005 (2019).

8. Wang, Z., Chong, Y., Joannopoulos, J. D. \& Soljacic, M. Observation of unidirectional backscattering-immune topological electromagnetic states. Nature 461, 772-775 (2009).

9. Rechtsman, M. C. et al. Photonic Floquet topological insulators. Nature 496, 196-200 (2013).

10. Khanikaev, A. B. et al. Photonic topological insulators. Nat. Mater. 12, 233-239 (2013).

11. Hafezi, M., Mittal, S., Fan, J., Migdall, A. \& Taylor, J. M. Imaging topological edge states in silicon photonics. Nat. Photon. 7, 1001-1005 (2013).

12. Kraus, Y. E., Lahini, Y., Ringel, Z., Verbin, M. \& Zilberberg, O. Topological States and adiabatic pumping in quasicrystals. Phys. Rev. Lett. 109, 106402 (2012)

13. Song, D. et al. Unveiling pseudospin and angular momentum in photonic graphene. Nat. Commun. 6, 6272 (2015).

14. Liu, J. L., Ye, W. M. \& Zhang, S. Pseudospin-induced chirality with staggered optical graphene. Light Sci. Appl. 5, e16094 (2016).

15. Diebel, F., Leykam, D., Kroesen, S., Denz, C. \& Desyatnikov, A. S. Conical diffraction and composite lieb bosons in photonic lattices. Phys. Rev. Lett. 116, 183902 (2016)

16. Lu, L. et al. Experimental observation of Weyl points. Science 349, 622-624 (2015).

17. Xu, S. Y. et al. Discovery of a Weyl fermion semimetal and topological Fermi arcs. Science 349, 613-617 (2015).

18. Lv, B. Q. et al. Experimental discovery of Weyl semimetal TaAs. Phys. Rev. X 5, 031013 (2015)

19. Dubcek, T. et al. Weyl points in three-dimensional optical lattices: synthetic magnetic monopoles in momentum space. Phys. Rev. Lett. 114, 225301 (2015).

20. Béché, A., Van Boxem, R., Van Tendeloo, G. \& Verbeeck, J. Magnetic monopole field exposed by electrons. Nat. Phys. 10, 26-29 (2013).

21. Berry, M. V. Quantal phase factors accompanying adiabatic. Chang. Proc. $R$. Soc. Lond. A 392, 45-57 (1984).

22. Bliokh, K. Y. et al. Theory and applications of free-electron vortex states. Phys. Rep. 690, 1-70 (2017).

23. Bliokh, K. Y., Rodríguez-Fortuño, F. J., Nori, F. \& Zayats, A. V. Spin-orbit interactions of light. Nat. Photon 9, 796-808 (2015).

24. Biener, G., Niv, A., Kleiner, V. \& Hasman, E. Formation of helical beams by use of Pancharatnam-Berry phase optical elements. Opt. Lett. 27, 1875-1877 (2002).

25. Marrucci, L., Manzo, C. \& Paparo, D. Optical spin-to-orbital angular momentum conversion in inhomogeneous anisotropic media. Phys. Rev. Lett. 96, 163905 (2006).

26. Karimi, E. et al. Generating optical orbital angular momentum at visible wavelengths using a plasmonic metasurface. Light Sci. Appl. 3, e167-e167 (2014).

27. Bliokh, K. Y., Niv, A., Kleiner, V. \& Hasman, E. Geometrodynamics of spinning light. Nat. Photon 2, 748-753 (2008).

28. Wang, S., Ma, G. \& Chan, C. T. Topological transport of sound mediated by spin-redirection geometric phase. Sci. Adv. 4, eaaq1475 (2018).

29. Bliokh, K. Y., Smirnova, D. \& Nori, F. Quantum spin Hall effect of light. Science 348, 1448 (2015)

30. Mecklenburg, M. \& Regan, B. C. Spin and the honeycomb lattice: lessons from graphene. Phys. Rev. Lett. 106, 116803 (2011).

31. Xu, X., Yao, W., Xiao, D. \& Heinz, T. F. Spin and pseudospins in layered transition metal dichalcogenides. Nat. Phys. 10, 343-350 (2014).

32. Zhang, Z. et al. Particlelike behavior of topological defects in linear wave packets in photonic graphene. Phys. Rev. Lett. 122, 233905 (2019).

33. Peleg, O. et al. Conical diffraction and gap solitons in honeycomb photonic lattices. Phys. Rev. Lett. 98, 103901 (2007).

34. Vicencio, R. A. et al. Observation of localized states in Lieb photonic lattices. Phys. Rev. Lett. 114, 245503 (2015)

35. Mukherjee, S. et al. Observation of a localized flat-band state in a photonic Lieb lattice. Phys. Rev. Lett. 114, 245504 (2015).

36. Xia, S. et al. Demonstration of flat-band image transmission in optically induced Lieb photonic lattices. Opt. Lett. 41, 1435-1438 (2016).

37. Ablowitz, M. J., Nixon, S. D. \& Zhu, Y. Conical diffraction in honeycomb lattices. Phys. Rev. A 79, 053830 (2009).

38. Efremidis, N. K., Sears, S., Christodoulides, D. N., Fleischer, J. W. \& Segev, M. Discrete solitons in photorefractive optically induced photonic lattices. Phys. Rev. E 66, 046602 (2002).

39. Fleischer, J. W., Segev, M., Efremidis, N. K. \& Christodoulides, D. N. Observation of two-dimensional discrete solitons in optically induced nonlinear photonic lattices. Nature 422, 147-150 (2003).

40. Basistiy, I. V., Bazhenov, V. Y., Soskin, M. S. \& Vasnetsov, M. V. Optics of light beams with screw dislocations. Opt. Commun. 103, 422-428 (1993).
41. Pereira, V. M., Castro Neto, A. H. \& Peres, N. M. R. Tight-binding approach to uniaxial strain in graphene. Phys. Rev. B 80, 045401 (2009).

42. Rechtsman, M. C. et al. Strain-induced pseudomagnetic field and photonic Landau levels in dielectric structures. Nat. Photon 7, 153-158 (2013).

43. Milićević, M. et al. Type-III and tilted Dirac cones emerging from flat bands in photonic orbital graphene. Phys. Rev. X 9, 031010 (2019).

44. Leanhardt, A. E. et al. Imprinting vortices in a Bose-Einstein condensate using topological phases. Phys. Rev. Lett. 89, 190403 (2002).

45. Van Mechelen, T. \& Jacob, Z. Photonic Dirac monopoles and skyrmions: spin-1 quantization [Invited]. Opt. Mater. Express 9, 95-111 (2018).

46. Dennis, M. R., King, R. P., Jack, B., O’Holleran, K. \& Padgett, M. J. Isolated optical vortex knots. Nat. Phys. 6, 118-121 (2010).

47. Pisanty, E. et al. Knotting fractional-order knots with the polarization state of light. Nat. Photon 13, 569-574 (2019).

48. Yan, Q. et al. Experimental discovery of nodal chains. Nat. Phys. 14, 461-464 (2018).

49. Lin, Q., Xiao, M., Yuan, L. \& Fan, S. Photonic Weyl point in a twodimensional resonator lattice with a synthetic frequency dimension. Nat. Commun. 7, 13731 (2016).

50. Lustig, E. et al. Photonic topological insulator in synthetic dimensions. Nature 567, 356-360 (2019).

51. Duca, L. et al. An Aharonov-Bohm interferometer for determining Bloch band topology. Science 347, 288 (2015).

52. Novoselov, K. S. et al. Unconventional quantum Hall effect and Berry's phase of $2 \pi$ in bilayer graphene. Nat. Phys. 2, 177-180 (2006).

\section{Acknowledgements}

This research is supported by the National Key R\&D Program of China under Grant (No. 2017YFA0303800), the National Natural Science Foundation (11922408, 91750204, 11674180), PCSIRT, and the 111 Project (No. B07013) in China. H.B. acknowledges support in part by the Croatian Science Foundation Grant No. IP-2016-06-5885 SynthMagIA, and the QuantiXLie Center of Excellence, a project co-financed by the Croatian Government and European Union through the European Regional Development Fund the Competitiveness and Cohesion Operational Programme (Grant KK.01.1.1.01.0004). D.L. is supported by the Institute for Basic Science in Korea (IBS-R024-Y1).

\section{Author contributions}

D.S., Z.C., and H.B. conceived the project. X.L. and S.X. performed the experiments and simulation. H.B., E.J., and D.L. performed the theoretical analysis. D.H.L., L.T., and J.X. participated in discussion. Z.C., H.B., and D.S. wrote the paper. All authors contributed to all aspects of this work.

\section{Competing interests}

The authors declare no competing interests.

\section{Additional information}

Supplementary information is available for this paper at https://doi.org/10.1038/s41467020-15374-x.

Correspondence and requests for materials should be addressed to D.S., H.B. or Z.C.

Peer review information Nature Communications thanks the anonymous reviewer(s) for their contribution to the peer review of this work.

Reprints and permission information is available at http://www.nature.com/reprints

Publisher's note Springer Nature remains neutral with regard to jurisdictional claims in published maps and institutional affiliations.

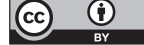

Open Access This article is licensed under a Creative Commons Attribution 4.0 International License, which permits use, sharing, adaptation, distribution and reproduction in any medium or format, as long as you give appropriate credit to the original author(s) and the source, provide a link to the Creative Commons license, and indicate if changes were made. The images or other third party material in this article are included in the article's Creative Commons license, unless indicated otherwise in a credit line to the material. If material is not included in the article's Creative Commons license and your intended use is not permitted by statutory regulation or exceeds the permitted use, you will need to obtain permission directly from the copyright holder. To view a copy of this license, visit http://creativecommons.org/ licenses/by/4.0/.

(C) The Author(s) 2020 